\title{
Enrichment of Poly Vinyl Chloride (PVC) Biological uses Through Sodium Chloride Filler, Density Functional Theory (DFT) Supported Experimental Study
}

\author{
A.M. Abdelghany ${ }^{1}$, M.S. Meikhail ${ }^{2}$, R. Hamdy ${ }^{2}$ \\ ${ }^{1}$ Spectroscopy Department, Physics Division, National Research Centre, 33 ElBehouth St., Doki, 12311, \\ Giza, Egypt \\ ${ }^{2}$ Physics Department, Faculty of Science, Mansoura University, 355165, Mansoura, Egypt \\ a.m_abdelghany@yahoo.com
}

\begin{abstract}
Samples of pristine poly vinyl chloride (PVC) and other samples of the same matrix containing a variable amount of inorganic sodium salt (sodium chloride $\mathrm{NaCl}$ ) were prepared via simple casting route in a dimethyl sulfoxide (DMSO) solvent were prepared. Prepared samples were characterized via $x$-ray diffraction (XRD) measurements and Fourier transform infrared spectrophotometric measurements (FTIR) in combination with computational density functional theory approaches (DFT) into account for a detailed picture of the possible reaction mechanisms within the polymeric matrices. All measurements were performed on the possible basis set. Local density approximate (LDA) and generalized Goertzel algorithm (GGA) was also tested. A compatibility between computed and experimental results suggested that physical interaction was preferred in such matrix and nominate use of this new material to be used in the medical field as a result of dioxin illumination.
\end{abstract}

Keywords: Poly Vinyl Chloride (PVC); Density Functional Theory (DFT); FTIR; XRD

Date of Submission: 30 September, 2018

DOI: 10.24297/jap.v14i3.7744

ISSN: 2347-3487

Volume: 14 Issue: 3

Journal: Journal of Advances in Physics

Website: https://cirworld.com

This work is licensed under a Creative Commons Attribution 4.0 International License. 


\section{Introduction}

A Long time ago polymeric materials are classified as macromolecules as a result of linked atoms or groups multiplication and repetition causing large size and higher molecular weights which may be homopolymer or copolymer (Linear, Branched or crosslinked) with variable characteristics [1,2]. Recently fillers added to bring a specific physical or chemical property for specific applications [3-7].

Lithium salts added to a polymer to form solid polymer electrolytes (SPE) with high specific energy, high energy density and other properties makes it of superior characteristics to be used in electrochromic devices and energy conversion systems such as fuel cells and batteries [4]. In such case polymers used as a binder to ensure the electrical exchange with electrodes or as a separator which separates anode and cathode and acts as a medium for the ions transported between anode and cathode throughout discharging and charging processes $[5,6]$.

Polyvinyl chloride (PVC) can be considered as a third widely produced polymeric material in the world formed by free radical polymerization of vinyl chloride and because of their cost and ease of processability in addition to their mechanical, physical and chemical characteristics [8]. In addition, PVC's are assumed to be a relatively low-cost biological material due to their chemical resistance through the addition of some modifiers and stabilizers.

PVC owe a superior insulating character combined with durability, flexibility and with a good resistant for aggressive environments so it is a suitable candidate for manufacturing of wiring, cables, housing material and in many medical instruments due to their safety, chemical stability and it was also non-toxic and owe a high biocompatibility with tissue/blood, their transparency makes it suitable for blood bags and intravenous containers manufacturing. Instruments made off PVC can also easily sterilized [9-11]. The main major issue is the production of dioxins which is a harmful chlorinated pollutant.

Fillers may be added to polymeric matrices to obtain a specific character for specific uses. Some have a synergistic effect; particles may reduce costs, shrinkage, molding cycle, increase thermal conductivity and decrease resistivity [12]. Conduction processes and mechanisms through materials may effect by the impurities or fillers and have a great role in polymeric semiconductors because it may reside in various sites or to become subsistent or interstitial of the polymer chain or reside at boundaries and diffuse favorably within amorphous regions forming charge transfer complexes and affect mobility via electron transfer from chain to chain $[13,14]$.

Density functional theory approach recently used to describe the ground state properties of materials including metallic, semiconducting, and insulating materials using functional of electron density. Therefore, it can be considered as a most well-known and adaptable computational physical techniques employing certain functions and basis sets [15].

The main aim of the presented work is to illustrate the role of inorganic sodium chloride salt as a filler within the poly vinyl chloride polymeric matrix in the structural and physical properties for the prevention of forming dioxins using experimental and computational approaches. In addition, to illustrate and characterize obtained materials containing sodium salt as a plasticizer.

\section{Materials and Methods}

Materials used include polyvinyl chloride (PVC) of high molecular weight supplied by Fluka Co., sodium chloride supplied by Sigma Aldrich Co. with a chemical formula shown in Figure (1) 


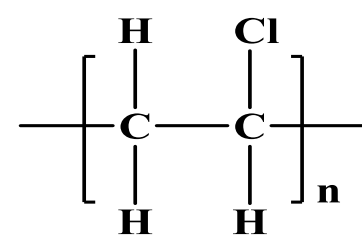

$2 \mathrm{D}$

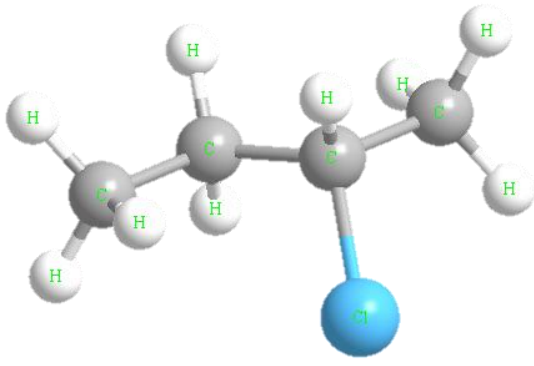

$3 \mathrm{D}$

Figure (1) 2D and 3D chemical structure of used PVC

The studied PVC films filled with inorganic filler $\mathrm{NaCl}$ were prepared via casting technique with a specific wt\% shown in the Table (1). DMSO was used as a common solvent to dissolve both polymer and sodium chloride under a constant vigorous stirring at room temperature to complete dissolution until a suitable viscous solution is formed. Obtained material prepared by the casting method in a glass dishes kept in an oven regulated at $60{ }^{\circ} \mathrm{C}$ for 3 days to ensure trace solvent evaporation. Resulting thin films husked from the Petri dishes and then kept in a vacuum desiccator until usage; suitable pieces from the films were cut for the measurements. The thickness of the obtained films was in the range of $\approx 0.05-0.2 \mathrm{~mm}$. Pre-calculated masses of both PVC and sodium salt were mixed according to:

$$
\mathbf{W}(\mathbf{w t} \%)=\frac{w_{f}}{w_{f}+w_{p}} \times \mathbf{1 0 0}
$$

Where $\mathrm{w}_{f}$ and $\mathrm{w}_{p}$ represent the weights of the filler and polymer, respectively. Sample notation and composition are listed in Table (1)

Table (1) sample notation and composition

\begin{tabular}{|c|c|c|c|c|c|c|}
\hline Sample & $\mathbf{S}_{\mathrm{Na}}$ & $\mathbf{S}_{\mathrm{Na} 1}$ & $\mathbf{S}_{\mathrm{Na} 2}$ & $\mathbf{S}_{\mathrm{Na} 3}$ & $\mathbf{S}_{\mathrm{Na} 4}$ & $\mathbf{S}_{\mathrm{Na}}$ \\
\hline PVC wt\% & 100 & 99 & 98 & 95 & 90 & 85 \\
\hline $\mathbf{N a C l} \mathbf{w t} \%$ & 0 & 1 & 2 & 5 & 10 & 15 \\
\hline
\end{tabular}

XRD diffraction was used to identify various crystalline or amorphous phases presented in the structures of

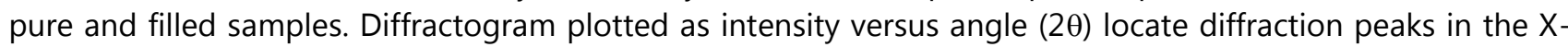
ray diffraction spectra. Measurements performed using PANalytical X'Pert PRO adopting $\mathrm{Cu}$ K $\alpha$ target using wavelength $\lambda=1.540 \AA$ and tube operating at $45 \mathrm{kV}-40 \mathrm{~mA}$ within the Bragg's angle (20) ranging between $5^{\circ}$ $80^{\circ}$. Single beam Fourier transform infrared spectrophotometer (Nicolet iS10, USA) in absorption mode operating with 32 runs and resolution $2 \mathrm{~cm}^{-1}$ at room temperature to collect spectral data with the range $4000-400 \mathrm{~cm}^{-1}$ to identify function group included within the material structure. Scanning electron microscope type (JEOL JSM-6510LV) was used to characterize surface morphology of prepared samples using $20 \mathrm{kV}$ source after coating of the sample with a thin layer of gold to minimize sample charging effect results from the incident electron beam.

All calculations were achieved using Gaussian 03 program [16] using DFT framework. Density functional calculations have been employed to ensure reaction mechanisms through an agreement between the experimental measured and theoretical calculated data. All assumed structures were optimized using Becke's three-parameter hybrid functional [17] introduced by Lee et al. [18] and developed correlation functional method (B3LYP) by Hay and Wadt with the electron core potential basis set WLANL2DZ [19]. 


\section{Results and Discussion}

\section{X-ray diffraction analysis}

X-ray diffraction (XRD) represents a powerful tool that can be used to identify the internal structural arrangement of solids. It can be used to differentiate between crystalline, amorphous and semicrystalline solids. In addition, it can be used to identify the presence of crystalline phase present in the structure. Figure (2) reveal XRD pattern of $\mathrm{NaCl}$ filler consists of sharp intense bands located at about $27^{\circ}, 32^{\circ}, 46^{\circ}, 54^{\circ}, 57^{\circ}, 66^{\circ}$, $76^{\circ}$ correspond to (111), (200), (220), (311), (222), (400), (420) planes of $\mathrm{NaCl}$.

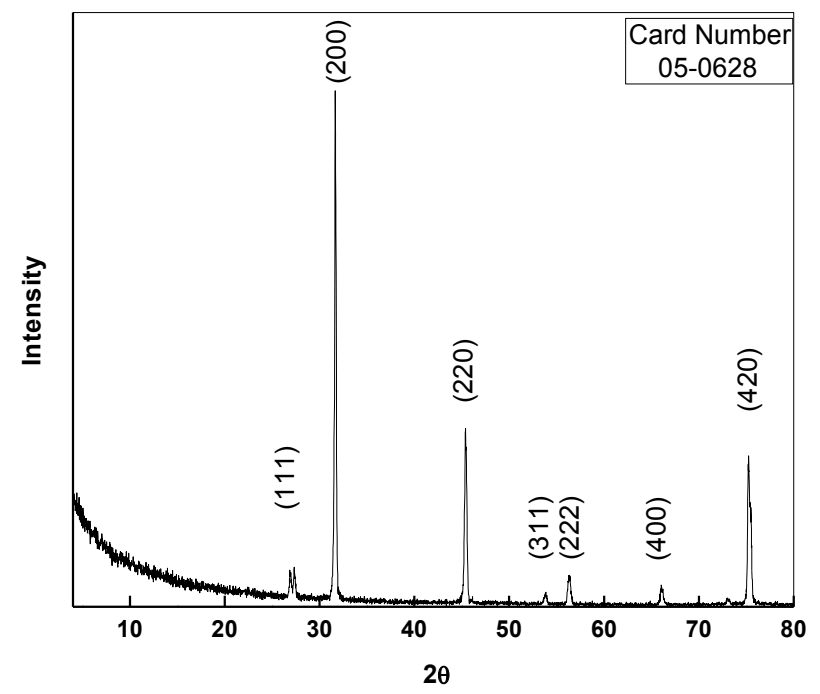

Figure (2): X-ray diffraction scans for $\mathrm{NaCl}$ salt

PVC generally exhibits an amorphous phase [20] XRD pattern of PVC shows a diffraction peak at $2 \Theta=18^{\circ}[21$, 22] while the role of the inorganic salt $(\mathrm{NaCl})$ can be investigated through full range measurement of the filled samples. Figure (3) reveals $\mathrm{x}$-ray diffractogram of pristine PVC and filled samples with $\mathrm{NaCl}$. Evaluation of the XRD diffraction patterns of pristine sodium chloride, PVC films and that of PVC containing variable amount of inorganic filler reveals presence no peak in the low salt content indicating complete solubility and homogenous distribution of the salt within the polymeric matrix. Further Increase in the sodium chloride content leads to the appearance of a sharp intensified peak at about $32^{\circ}$ belong to attributed to the presence of sodium salt separated phase within the polymeric matrix belong to (200) plane of $\mathrm{NaCl}$. Multiple peaks with lower intensities appear at the position shown in Figure (3) attributed to (220), (311), (222) and (420) of sodium chloride respectively. It was observed also that the amorphous halos around $2 \theta \approx 26$ are less intense in the filled PVC films in comparison with that present in the pattern of pristine PVC films which means that addition of $\mathrm{NaCl}$ decrease the of crystalline phase in PVC filled samples in agreement with the results reported by Uma et al. [23] and pointing to the formation of multiple phases in the new material composed of combined semicrystalline and amorphous phases [24].

XRD pattern of a polymeric matrix containing the successive amount of sodium salt shown in Figure (3) reveals maintenance of characteristic amorphous halos around $18^{\circ}$ and $25^{\circ}$. Sample containing more than $1 \mathrm{wt} . \% \mathrm{NaCl}$ show appearance of the sharp band located at about 32 and 57 characterize the cubic structure of sodium chloride salt. Further increase in salt concentration leads to an increase in peak sharpen and hence sample crystallinity result from presence phase separated sodium salt polymeric matrix and forms lamellar composite structure. 


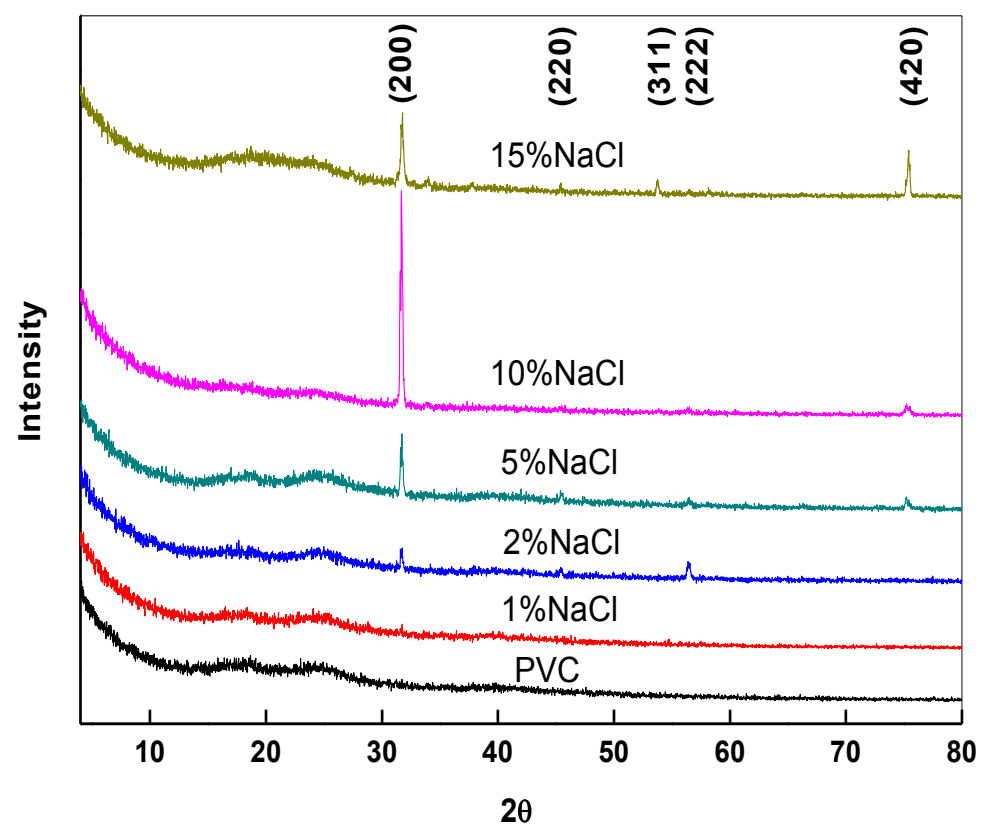

Figure (3): X-ray diffraction scans of pure PVC and PVC filled with different concentrations of $\mathrm{NaCl}$.

\section{Fourier transform infrared analysis (FTIR)}

FTIR spectra of $\mathrm{NaCl}$ Figure (4) shows the appearance of $\mathrm{NaCl}$ characteristic band located at nearly $619 \mathrm{~cm}^{-1}$ in addition to two sharp bands located at 1635 and $3440 \mathrm{~cm}^{-1}$ assigned to bound water and oH groups that may result from hygroscopic nature of studied salt without any evidence for the presence of any other function groups. Figure (5) shows FTIR spectra of virgin PVC and other samples that filled with a different mass fraction of sodium salt $(\mathrm{NaCl})$.

All spectral data reveals nearly same behavior with a minor variation in intensity without any evidence for the formation of new bands. The spectra characterized by a broad hump originally located at about $3500 \mathrm{~cm}^{-1}$, their area and intensity increases with increasing $\mathrm{NaCl}$ content.

The following features were also observed:

- $\quad$ Two successive sharp bands located at 2971, $2911 \mathrm{~cm}^{-1}$.

- Three sharp intense bands in the mid-region between 1436: $1252 \mathrm{~cm}^{-1}$.

- $\quad$ Two split bands with variable intensity sensitive to $\mathrm{NaCl}$ content change located between 1000:1400 $\mathrm{cm}^{-1}$.

- $\quad$ Sharp split band located at about $612 \mathrm{~cm}^{-1}$.

Obtained data and their assignment can be summarized in Table (2). 


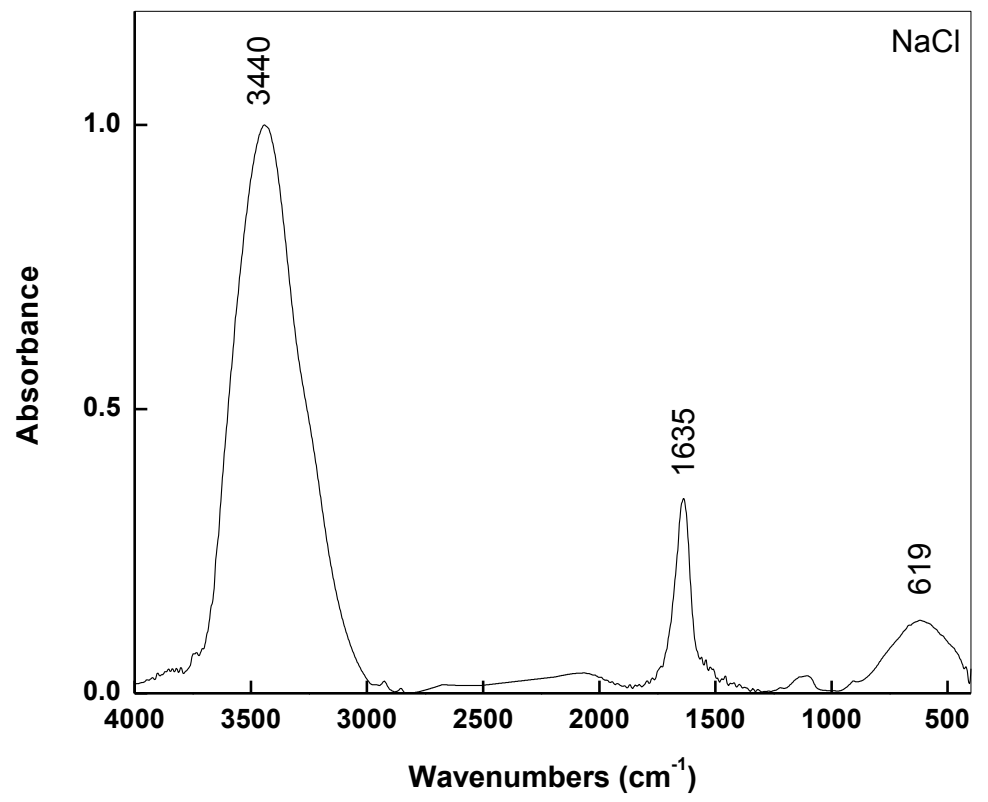

Figure (4): FT-IR spectrum of $\mathrm{NaCl}$ salt.

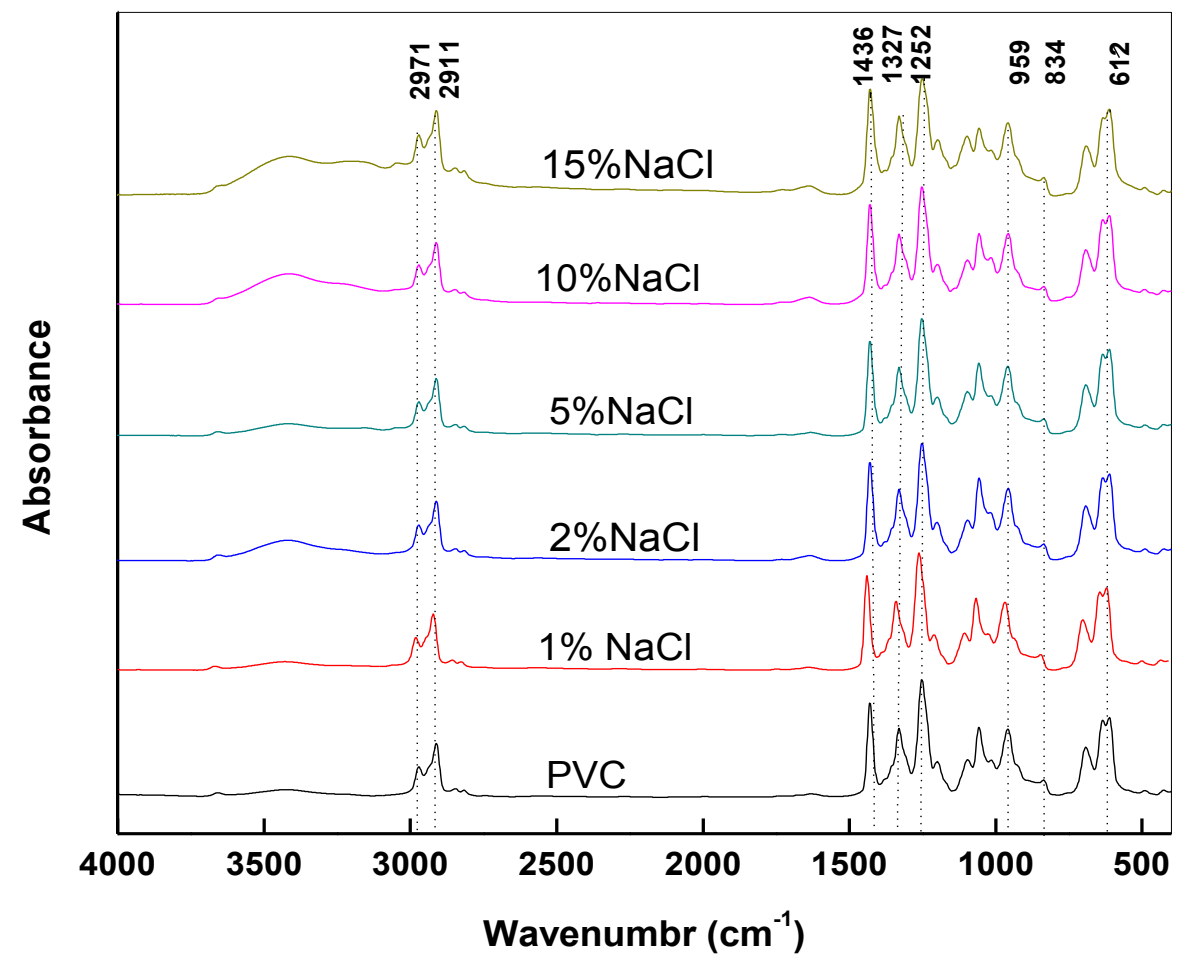

Figure (5): FT-IR spectra of PVC with different concentrations of $\mathrm{NaCl}$ salt. 
Table (2): Peak position and assignment for the vibrational modes observed in PVC.

\begin{tabular}{cc}
\hline Description of vibration & Wavenumbers $\left(\mathbf{c m}^{\mathbf{- 1}}\right)$ \\
\hline$-\mathrm{CH}$ asymmetric stretching & 2971 \\
$-\mathrm{CH}$ stretching & 2911 \\
$\mathrm{CH}_{3}$ stretching & 1436 \\
$-\mathrm{CH}_{2}$ deformation & 1327 \\
$\mathrm{C}-\mathrm{H}$ rocking & 1252 \\
Trans C-H wagging & 959 \\
$\mathrm{C}-\mathrm{Cl}$ stretching & 834 \\
cis C-H wagging & 612 \\
\hline
\end{tabular}

\section{Density Functional Theory (DFT) calculations}

Density function theory approach (DFT) represent a class of computational calculation used for a variety of purposes including but not limited to structure and energy optimization frequency and charge calculation in addition to the thermal variable. (DFT) employ a number of basis sets and functions for data application. In our study only local density approximate (LDA) and generalized Goertzel algorithm (GGA) or sometimes called generalized gradient approximation. Figure (6) shows two-dimension (2D) and 3-dimension (3D) structure respectively of the studied sodium salt, polymeric matrix of poly vinyl chloride (PVC) and all possible suggested interaction mechanisms including chemical and physical interactions indicated by $T_{1}, T_{2}, T_{3}$ and $T_{4}$ depending on the position of sodium atom attack.

\section{Mechanism}

2D<smiles>CC(N)C(C)Cl</smiles>

T2

T1<smiles>CCC(C)([N])Cl</smiles>

3D

Interaction
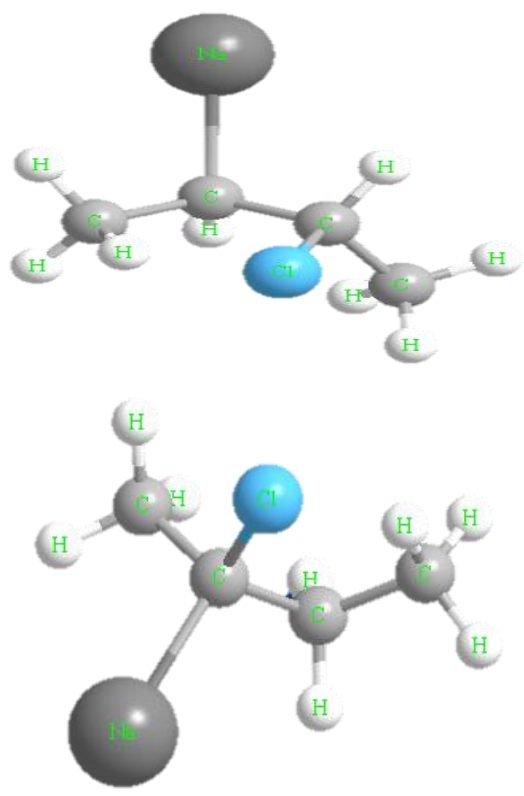

\section{Chemical}

\section{Chemical}


T3<smiles>CCC(C)Cl</smiles>

T4<smiles>CCC(C)[N+]</smiles>

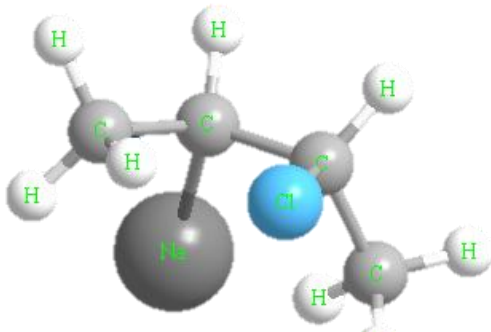

Chemical

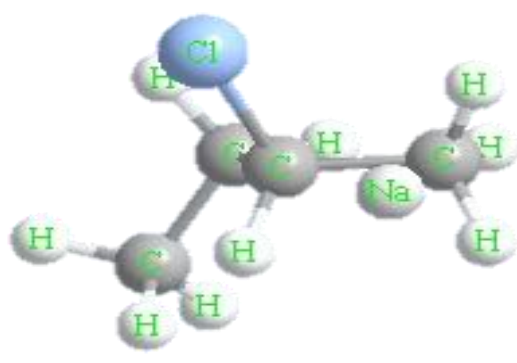

Physical

Figure (7) 2D and 3D suggested reaction mechanisms between polymer (PVC) and inorganic filler $(\mathrm{NaCl})$

Figure (8) shows both experimental and theoretical calculated infrared spectra of virgin PVC sample using two different approximation model a-GGA-PW91 and b-LDA-PWC. It was noticed that approximation give more accurate IR spectrum which is in agreement with the obtained experimental data with a percent compatibility not less than $91.2 \%$ and with variable intensities that may result from matrix effect since the studied model was consists of one monomer.

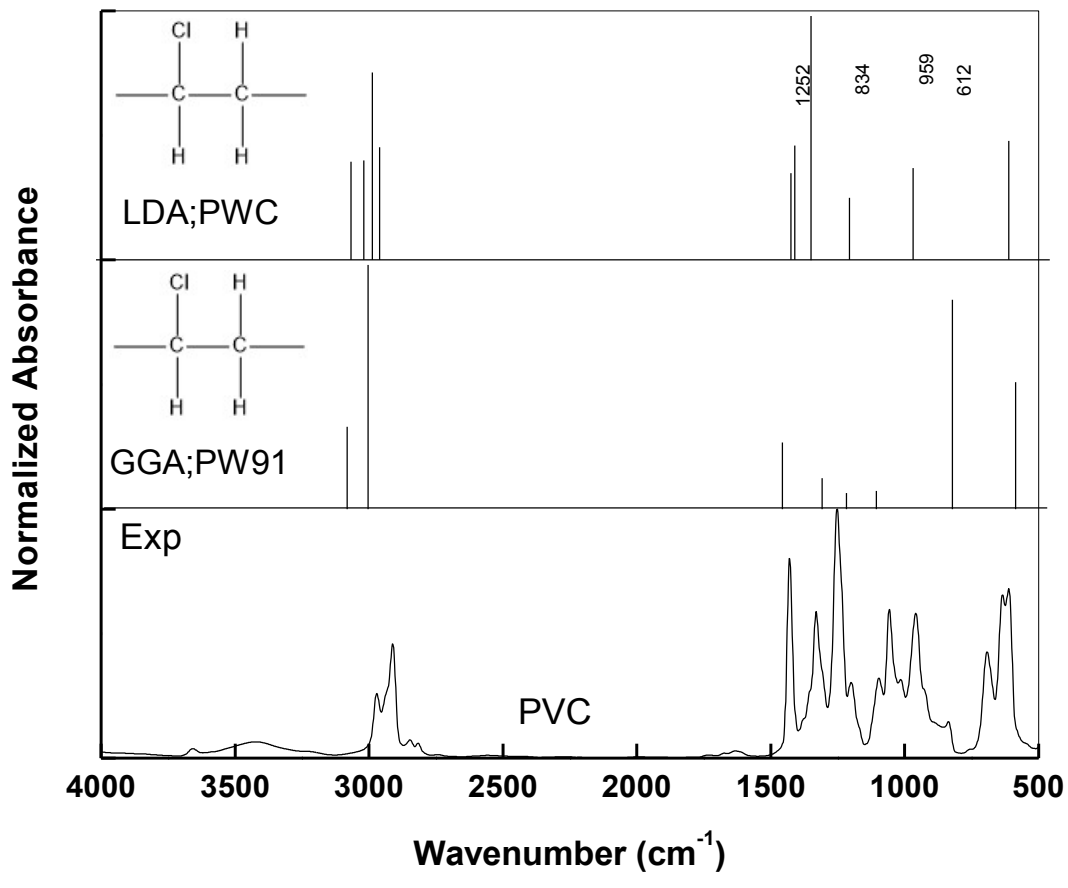

Figure (8) experimental and theoretical FTIR for PVC 


\section{a. Chemical interaction of $\mathrm{NaCl}$ with PVC}

Figure (9) shows the different trail of suggested reaction mechanisms between polymer and sodium salt using a single approximation (GGA-PW91). It was obvious to conclude that the approximation $\left(T_{2}\right)$ is forbidden or cannot be calculated using such function while (T1) and (T3) are preferred and give a high percentage of compatibility $93 \%$ and $89 \%$. Experimental data for the sample that contain $10 \%$ sodium was taken for comparison because all spectral data considered to be repetitive but with different intensities. The sample that contains $10 \% \mathrm{NaCl}$ having an intensified spectra.

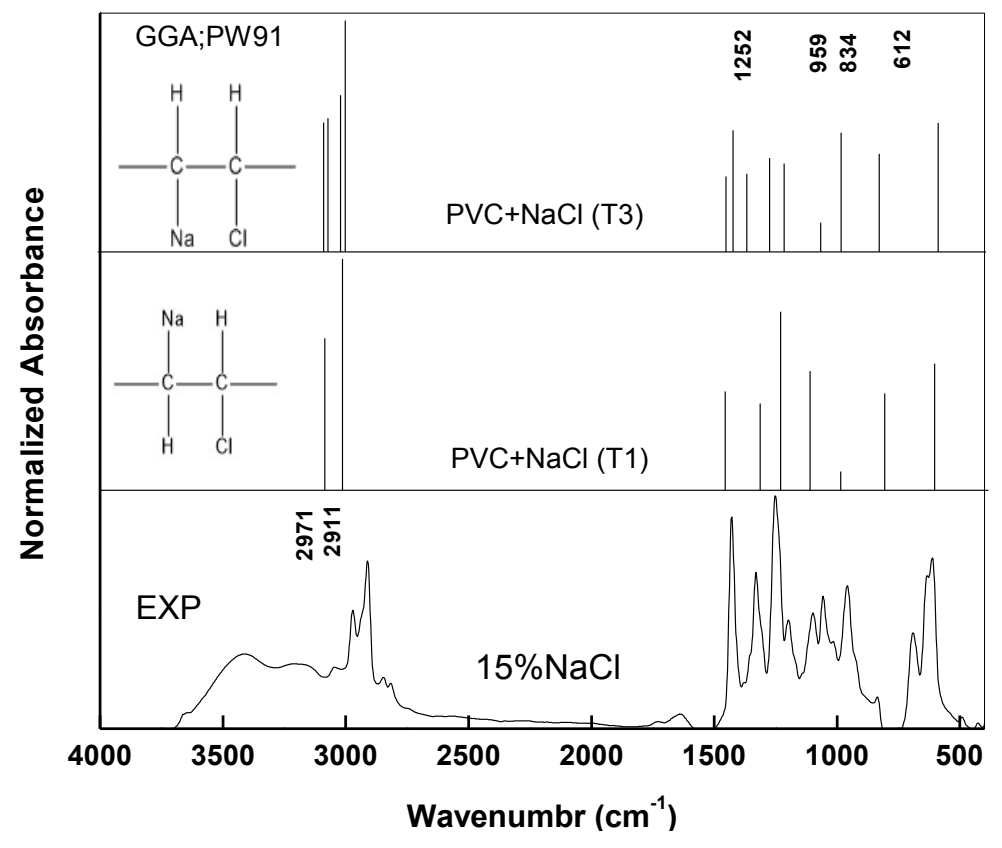

Figure (9) experimental and theoretical FTIR for PVC+ $\mathrm{NaCl}$.

Figure (10) shows the different trail of suggested reaction mechanisms between polymer and sodium salt using a single approximation (LDA-PWC).

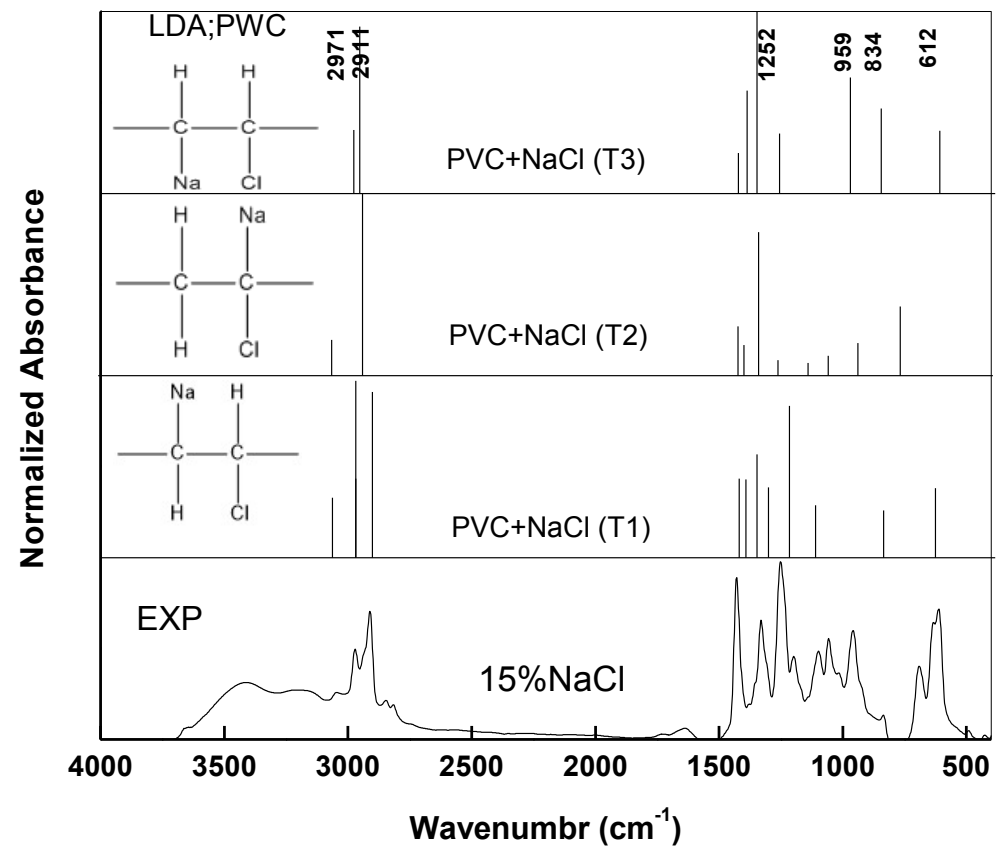

Figure (10) experimental and theoretical FTIR for PVC $+\mathrm{NaCl}$. 


\section{b. Physical interaction of $\mathrm{NaCl}$ with PVC}

Figure (11) reveals the proposed structure evaluated for the physical interaction between the polymeric matrix and sodium salt and indicate as $\left(\mathrm{T}_{4}\right)$. It was obvious to conclude that this assumption given is the most promising results due to the high compatibility between theoretical and experimental results nearly for all PVC characteristic bands and may be attributed to the high stability and bonding of PVC that prevent or decreases the probability of any chemical interaction within the polymer network.

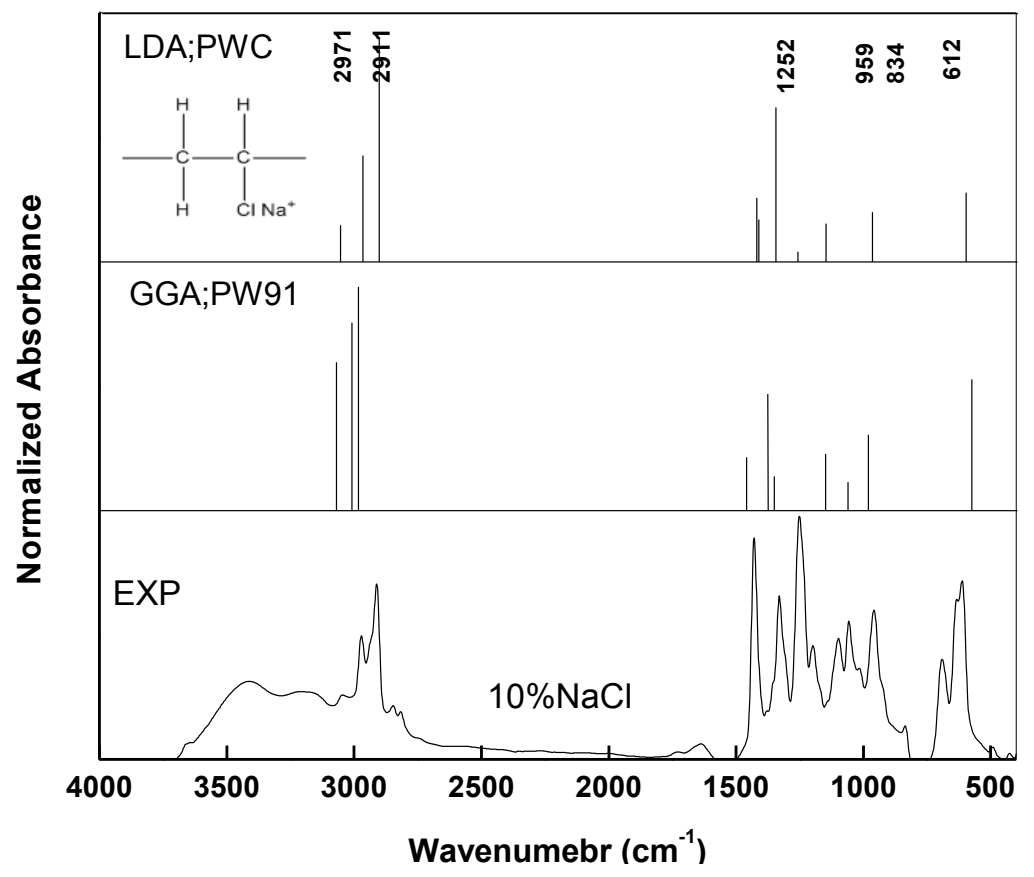

Figure (11) experimental and theoretical FTIR for PVC+NaCl.

\section{Conclusions}

Proposed samples of as received high molecular weight poly vinyl chloride (PVC) in addition to other samples containing a variable mass fraction of sodium chloride $\mathrm{NaCl}$ were synthesized via ordinary casting route. X-ray diffraction (XRD) measurements support the nature of crystalline sodium chloride and show the presence of crystalline bands at the specific position in the heavy filled samples attributed to the presence of crystalline sodium chloride separated phase. Fourier transform infrared spectrophotometric measurements (FTIR) in combination with computational density functional theory approaches (DFT) reveal the maintenance of PVC characteristic bands and does not show the appearance of any new peak pointing to the physical interaction between salt and polymeric matrices. Computational data perform the proposed possible reaction mechanisms and gives the percentage compatibility between experimental and computed spectral data via both local density approximate (LDA) and generalized Goertzel algorithm (GGA). Obtained data nominate use of synthesized new material to be used in the medical field as a result of dioxin illumination.

\section{References}

1. L.A. Utracki, Polymer Alloys and Blends, Carl Hanser, Munich, FRG, 1990.

2. L.H. Sperling, Introduction to Physical Polymer Science, John Wiley and Sons, Inc.

3. J.Y. Kim, S.H. Kim, Solid State Ionics 124 (1999) 91.

4. S.S. Sekhon, N. Arora, S.A. Agnihotry, Solid State Ionics 136-137 (2000) 1201.

5. S. Ramesh, C.W. Liew, Polymer Bulletin, 70 (2013) 1277. 
6. S. Tripathi, A. Gupta, M. Kumari, Bulletin of Materials Science, 35 (2012) 969.

7. S. Ramesh, M.F. Chai, Mater. Sci. Eng. B 139 (2007) 240.

8. W.V. Titow, PVC technology, $4^{\text {th }}$ ed., Springer, 1986.

9. M. Chanda, S.K. Roy, Plastics technology handbook, CRC Press, 2006.

10. J.W. Nicholson, The chemistry of polymers royal society of chemistry paperbacks, London, 1991.

11. J. Emsley, The consumer's good chemical guide: a Jargon-free guide to controversial chemicals, WH Freeman, 1994.

12. A. Moustafa, A. Faizalla, B. Abd El Hady, Journal of Applied Polymer Science, 67 (1998) 637.

13. R. Mukhopadhyay, S.K. De, S. Basu, Journal of Applied Polymer Science, 20 (1976) 2575.

14. S. Chand, P. Mehendru, Journal of Physics D: Applied Physics, 19 (1986) 857-859.

15. Kohn, W. and L.J. Sham, Physical Review, 140(4A)(1965)A1133.

16. M. J. Frisch, G.W. Trucks, H. B. Schlegel, G. E. Scuseria, M. A. Robb, Gaussian 03, Revision A.1, Gaussian, Inc., Pittsburgh PA, 2003.

17. D. Becke, J. Chem. Phys., 98 (1993) 5648.

18. W. Lee, R. Yang, G. Parr, Physical Review B, 37 (1988) 785.

19. P. J. Hay, W. R. Wadt, Journal of Chemical Physics, 82 (1985) 270.

20. S. Sejal, Q. Anjum, D. Singh, N. L. Singh, K. P. Singh, V. Shrinet, Indian Journal of Pure and Appllied Physics, 46(2008)439-442.

21. S. Rajendran, M.Ramesh Prabhu, M. Usha Ran, International Journal of electrochemical Sciences, $3(2008) 282-290$.

22. S. Rajendran, R. S. Babu, M. U. Rani, Bulltin of Material Science, 3(2011)1525-1530.

23. T. Uma, T. Mahalingam, U. Stimming, Journal of Materials Science, 39(2004)2901.

24. J. Li, X. Huang, K. Chen, Journal of Electrochemical Society, 147(7) (2000)2653. 